\title{
OBSERVATIONAL AND THEORETICAL MODELING OF NUTATION
}

\author{
P. M. MATHEWS \\ Department of Physics, University of Madras, India
}

AND

T. A. HERRING

Department of Earth, Atmospheric and Planetary Sciences, MIT, USA

\section{Observational Nutation Results: IERS 1996 Conventions series}

The IERS nutation series was developed as a nutation series which would match observations and could be used with geodetic systems which have sensitivity to nutations but are not able to determine accurately corrections to the IAU-1980 nutation series. After experimentation with different methods for parameterizing the nutation estimation problem, the best fit to the VLBI observed nutation angle data was found by estimating selected coefficients in the Mathews et al. (1991a) nutation response function (see equation (1) below), along with other parameters to be discussed below. The estimation of the response function coefficients was also found to account for mantle anelasticity and the loading part of the ocean tide correction and therefore no corrections were applied for these effects. Their contributions should be absorbed into the estimated response coefficients. These initial analyses also showed: setting the inner-core response to zero yielded better fits to the nutation angle data than the Mathews et al. (1991b) theoretical response and resonance frequency; the amplitude of the RFCN free mode appeared to be time dependent; and the prograde annual nutation was significantly affected by the S1 thermal tide which is not proportional to the $S_{1}$ gravitational potential.

The data set used to estimate the IERS conventions series was the GSFC nutation angle data set with 2040 pairs of adjustments to the IAU 1980 nutations in obliquity and longitude spanning the interval between 1979 and 1995. Here we also compare the IERS conventions series with a US Naval Observatory analysis of VLBI data between 1979 and August 1997. This data set consists of 2152 pairs of nutation angle estimates and extends by 1.8 years the interval covered by the GSFC data set. It also contains less pre-1984 data than the GSFC data set which accounts for the smaller increase in total number of observations than would be expected for the additional time interval covered.

The parameters estimated to model the GSFC data were $R, R^{\prime}$ and the retrograde Free Core Nutation (FCN) resonance strength $R_{F C N}$ and resonance frequency $\sigma_{F C N}$. All these parameters were estimated as complex quantities. In addition, we estimated linear rates of change of the longitude and obliquity, the in- and out-of-phase components of prograde annual nutation, and free terms with the frequency of the RFCN at two year intervals after 1984. One free term was estimated for all the data before 1984 because the density and quality of the data prior to 1984 was not sufficient to support the 2-year estimates. The fundamental arguments of Simon et al. (1994) were used with the rigid-Earth nutation series, along with planetary nutations, of Souchay and Kinoshita (1996) (electronic version of October 23, 1995). An additional correction was applied due to annual modulation of the geodetic precession (Fukushima, 1991).

Table 1 gives the differences for the largest nutations between the IERS series and the IAU1980 series and the analysis of the USNO data set. In general, the agreement between the USNO analysis and the IERS series is consistent with the uncertainties of the former. One exception to this agreement is the 13.66 day prograde nutation where the USNO analysis value differs from the GSFC value by $32 \mu$ as. This difference appears to be due to different treatment of the ocean- 
driven diurnal and semidiurnal rotation changes by the two groups. Recent GSFC analyses are more consistent with the USNO analysis suggesting that a common model in now in use.

\section{Theoretical Models}

Predictions of the amplitudes of the circular components of nutation are based on theoretical treatments of the nutations of an idealized (oceanless, elastic) ellipsoidal Earth model with axial symmetry under excitation by the spectral components of the tide generating potential (TGP), and of the contributions to the amplitudes from ocean tides, mantle anelasticity, and other mechanisms (e.g., electromagnetic and topographic couplings of the fluid outer core (FOC) to the mantle and to the solid inner core (SIC)).

A solid accomplishment from recent work is the attainment of microarcsecond ( $\mu$ as) level accuracy in the rigid Earth nutation series, needed for the understanding of nutation observations at current levels of precision. (The nutation of a rigid Earth is determined by the TGP and the Earth's dynamical ellipticity $e \equiv(C-A) / A)$. Much effort has also gone into the modeling of deviations from hydrostatic equilibrium structure for the ellipsoidal Earth and into the computation of their effects on nutation.

Progress during the last several years in respect of other aspects of the theory has not, however, been significant. In regard to mantle anelasticity, the most commonly used models are based on a constant value of mantle $Q$ from the 1 second reference period of PREM to some longer period $T_{s}$ (say, $200 \mathrm{sec}$ ) and a $T^{-\alpha}$ dependence of $Q$ for the range of periods from $T_{s}$ to tidal. The values to be assigned to $T_{s}$ and $\alpha$ are not well determined yet, and recent computations of anelasticity contributions to nutation amplitudes, using various plausible sets of values of $T_{s}$ and $\alpha$, follow the approach of Wahr and Bergen (1986). Estimation of the contributions from ocean tidal loading has not benefited much from the substantial improvements stemming from the use of TOPEX/POSEIDON data in the modeling of ocean tides, because no significant improvement has resulted therefrom in the precision of estimates of the amplitude (relevant to nutation) of the degree 2 tesseral part of the ocean tide function. The contributions from core-mantle boundary couplings will be considered further below.

\subsection{RIGID EARTH NUTATION THEORY:}

The amplitudes of spectral components of the rigid Earth's nutation, computed to $0.1 \mu$ as accuracy by independent methods by Bretagnon et al. (1997), Souchay and Kinoshita (1997), and Roosbeek and Dehant (1997), are in almost complete agreement. (Some of these have out-of-phase parts-a recent discovery, see Williams (1994).) The process of evaluation of the rigid Earth amplitudes involved consideration of a number of direct and indirect planetary effects, the effects of nutation on the TGP, the " $J_{2}$ tilt" and triaxiality effects, etc. These play an essential role in the accurate determination of the Earth's dynamical ellipticity $e$ from the observed rate of precession of the equator. The values obtained for $e$ by the different groups (and reflected in the respective nutation series) are in close agreement, and is just about $0.006 \%$ higher than the value used in the IAU 1980 theory of nutation. The new rigid Earth series have been used by Mathews and Buffett (unpublished, 1997) and by Dehant and Defraigne (1997) in their very recent computations for the deformable Earth with nonhydrostatic structure (see below).

\subsection{ELLIPSOIDAL EARTH MODEL:}

The $e$ determined as indicated above is over 1\% larger than the value for the ellipsoidal Earth model constructed (from PREM, for instance) under the assumption of a hydrostatic equilibrium structure; and the dynamical ellipticity $e_{f}$ of the fluid core as inferred from the free core nutation (FCN) resonance period of about 430 days needed to explain the observed retrograde annual nutation amplitude (Herring et al., 1986, Gwinn et al., 1986) is nearly 5\% larger than the hydrostatic equilibrium value. Such nonhydrostatic structure was taken account of in the analytic nutation theory of Mathews et al. (1991a,b) by simply using the values thus inferred for these ellipticity parameters. In recent work following this approach (Mathews and Buffett, unpublished, 1997), the ellipticity contributions to deformability parameters that also enter the analytical theory have been taken into account, along with the effects of the irrotational part of the core fluid flow on 
these parameters (Buffett et al., 1993). In the treatment of nutation theory along the lines of Wahr (1981), deviations from hydrostatic equilibrium have to be introduced by modifying the sets of surfaces of equal density/rheology/potential throughout the mantle as well as the boundary surfaces (CMB, $670 \mathrm{~km}$ discontinuity). Defraigne (1996) has used the viscous forces associated with mantle convection flows to produce the nonhydrostatic values $e$ and $e_{f}$ (or rather, an equivalent flattening of the CMB) referred to above, as well as agreement with certain other types of observational data; nutation amplitudes based on this model have been computed by Dehant and Defraigne (1997). A nonhydrostatic structure having the desired $e_{f}$ but not derived from a dynamical model has been employed by Schastok (1997) in computing nutation amplitudes. The amplitudes computed by these authors for a few of the most important nutations are compared in Table 1 with recent observational estimates.

TABLE 1. Theoretical predictions and observational estimates of nutation amplitudes: Differences from IAU 1980 in mas

\begin{tabular}{ccrrrrr} 
Period & & Theoretical & & predictions & IERS1996 & Observed \\
(Days) & & DD & \multicolumn{1}{c}{$\mathrm{S}$} & $\mathrm{MB}$ & $\mathrm{H}$ & $\mathrm{H}$ \\
13.66 & $i p$ & -0.18 & -0.14 & -0.060 & -0.145 & $-0.113 \pm 0.006$ \\
& $o p$ & -0.14 & -0.17 & -0.023 & -0.130 & $-0.139 \pm 0.006$ \\
-13.66 & $i p$ & -0.02 & -0.02 & -0.023 & -0.019 & $-0.014 \pm 0.006$ \\
& $o p$ & 0.01 & 0.02 & 0.007 & 0.015 & $0.032 \pm 0.006$ \\
182.62 & $i p$ & 0.67 & 0.67 & 0.644 & 0.606 & $0.593 \pm 0.006$ \\
& $o p$ & 0.45 & 0.49 & 0.554 & 0.510 & $0.514 \pm 0.006$ \\
-182.62 & $i p$ & -0.02 & -0.02 & -0.040 & -0.065 & $-0.050 \pm 0.006$ \\
& $o p$ & 0.05 & 0.07 & 0.034 & 0.047 & $0.048 \pm 0.006$ \\
365.26 & $i p$ & -0.02 & 0.07 & 0.036 & -0.012 & $-0.017 \pm 0.006$ \\
& $o p$ & -0.03 & -0.02 & -0.022 & -0.124 & $-0.138 \pm 0.006$ \\
-365.26 & $i p$ & -1.98 & -1.94 & -1.984 & -1.978 & $-1.976 \pm 0.013$ \\
& $o p$ & 0.12 & 0.11 & -0.298 & -0.322 & $-0.344 \pm 0.006$ \\
6798.38 & $i p$ & -0.03 & 0.07 & 0.056 & -0.099 & $-0.038 \pm 0.027$ \\
& $o p$ & 0.01 & 0.10 & 0.073 & 0.054 & $0.062 \pm 0.006$ \\
-6798.38 & $i p$ & -2.34 & -2.62 & -2.740 & -2.749 & $-2.782 \pm 0.027$ \\
& $o p$ & -0.86 & -1.01 & -1.324 & -1.490 & $-1.524 \pm 0.006$
\end{tabular}

DD: Dehant and Defraigne (1997), S: Schastok (1997), MB: Mathews and Buffett (unpublished, 1997), H: Estimates by Herring discussed above from VLBI data; ip: in-phase, op: out-of-phase. All the predictions include the respective authors' estimates of contributions from anelasticity and ocean tides. Ocean tidal current effects are ignored by MB, who include, however, Buffett's (1992) estimates of CMB electromagnetic coupling contributions. The sigmas are computed such that $\chi^{2} / f$ of the fit to the nutation angle data is unity where $f$ is the degrees of freedom.

The near agreement of the predictions for the retrograde annual in-phase amplitude with the observed value in all three cases is the result of adjusting $e_{f}$ or the equivalent CMB flattening to achieve such agreement. For a number of other frequencies, differences among the three predictions are rather large (tens to hundreds of $\mu \mathrm{as}$ ), presumably due to differences in various aspects of the modeling. It is worth noting, however, that inclusion of the CMB electromagnetic coupling effects (Buffett, 1992) in the computations of Mathews and Buffett (unpublished, 1997) has helped not only to nearly eliminate the discrepancy of $\approx 0.45$ mas found between the other authors' results for the retrograde annual out-of-phase amplitude and the observational estimate, but also to reduce the discrepancies in the 18.6 year amplitudes (both in-phase and out-of-phase). Nevertheless, overall agreement with the observational estimates cannot be considered satisfactory for any of the theoretical trcatments presented. 


\subsection{INVERSE MODELING:}

Least squares fitting of a formula derived from general theory to nutation amplitudes estimated from observations yields a "best fit" formula that can be used for prediction of celestial pole offsets, and the estimates obtained for parameters in the formula by the fitting procedure may provide useful constraints on Earth models. Resonance formulae for the transfer function $T(\sigma)$ from the nutation amplitude $\tilde{\eta}_{\text {rigid }}(\sigma)$ for a rigid Earth to $\tilde{\eta}(\sigma)$ for a deformable Earth are most convenient for such inverse modeling.

The resonance formula due to Mathews et al. (1991a), good for an oceanless Earth, is

$$
T(\sigma) \equiv \frac{\tilde{\eta}(\sigma)}{\tilde{\eta}_{\text {rigid }}(\sigma)}=R+R^{\prime}(1+\sigma)+\sum_{\alpha} \frac{R_{\alpha}}{\sigma-\sigma_{\alpha}}
$$

wherein $\sigma$ is the tidal excitation frequency, and the sum is over the relevant normal modes: the Chandler wobble (CW), the retrograde free core nutation (FCN), and the inner-core-induced prograde free core nutation (PFCN). (The frequency dependence of the ocean tide response to the TGP complicates the frequency dependence of $T(\sigma)$ for an Earth with oceans.) Sum rules following from the general theory connect $R$ and $R^{\prime}$ to the resonance frequencies $\sigma_{\alpha}$ and coefficients $R_{\alpha}$. (All frequencies are in cycles per sidereal day (cpsd)). One may obtain the following reduced resonance formula by using these sum rules:

$$
T\left(\sigma, e \mid e_{0}\right) \equiv \frac{\tilde{\eta}(\sigma, e)}{\tilde{\eta}_{\text {rigid }}\left(\sigma, e_{0}\right)}=\left(e_{0}-\sigma\right)\left(\frac{e}{e_{0}}\right)\left\{\frac{1}{1+e}+(1+\sigma) \sum_{\alpha} \frac{N_{\alpha}}{\sigma-\sigma_{\alpha}}\right\}
$$

where $e_{0}$ is the value used for $e$ in the rigid Earth nutation series employed, and the possibility is left open that the actual $e$ for the Earth might be slightly different. Estimates obtained for the resonance parameters $N_{\alpha}$ and $\sigma_{\alpha}$ may be used, in turn, to estimate or constrain ellipticity, deformability, and other basic parameters of the Earth and its core regions, since analytical expressions in terms of such basic parameters which give close approximations to the $N_{\alpha}$ and $\sigma_{\alpha}$ are available.

A least squares fit (carried out by Mathews) of the reduced resonance formula to VLBI estimates of the in- and out-of-phase amplitudes of 16 prograde-retrograde pairs of nutations and of the precession rate (obtained by Herring), has yielded estimates for the first time for a number of parameters besides the FCN period. The results given below were obtained from a fit done after removal of estimated anelasticity, ocean loading, and CMB electromagnetic coupling contributions from the nutation amplitude data before fitting. They may therefore be compared directly to values computed for standard Earth models. (The CMB coupling correction significantly improved the fit.) The estimates obtained were: $e=(32845520 \pm 16) \times 10^{-10}$; the coefficient of the PFCN resonance $N_{P F C N}=(191 \pm 37) \times 10^{-6}$, indicating a firm detection of this inner-core-induced mode with $\sigma_{P F C N}=-0.99882 \pm 0.00095$ corresponding to a best estimate of 844 days (with a rather large uncertainty) for the PFCN period in space; $N_{F C N}=(5155 \pm 14) \times 10^{-5}$; FCN period $=-433.0 \pm 0.3$ days, close to the period inferred by Herring et al. (1986) from the retrograde annual nutation alone; Chandler wobble period $=390.9 \pm 0.9$ days. Inferences about the Earth's properties may be drawn from the estimates with the aid of approximate analytical expressions for the resonance parameters: $\sigma_{C W}=-e N_{C W}=\left(A / A_{m}\right)(e-\kappa) ; \sigma_{F C N}=-1-\left(A / A_{m}\right)\left(e_{f}-\right.$ $\beta) ; N_{F C N}=\left(A_{f} / A_{m}\right)(1-\gamma / e) ; \sigma_{P F C N}=e_{s}\left\{1-\left(P+Q \rho_{f}^{I C B} e_{s}\right)\left(1-R \rho_{f}^{I C B}\right)\right\}$, where $P, Q, R$ are certain parameters of a standard Earth, $A, A_{m}, A_{f}$ are moments of inertia, and $e, e_{f}, e_{s}$ dynamical ellipticities, of the Earth and of its mantle, fluid outer core, and solid inner core regions (indicated by subscripts $m, f, s$, respectively), and $\kappa, \beta, \gamma$ are deformability parameters (see Mathews $e t$ al., 1991a). In particular, the 844 day period found for the PFCN, which contrasts with the computed value (476 days) based on PREM under hydrostatic equilibrium, would suggest a lower ellipticity for the inner core or a larger density contrast across the inner core boundary (ICB) than in PREM, apart from a possible role for magnetic coupling across the ICB. The estimates for the other resonance parameters confirm the already known excess ellipticity of the fluid core as well as of the whole Earth; other small deviations from PREM (hydrostatic) of $\kappa$ and $\gamma$ are indicated, but it is not clear that they are statistically significant.

The postfit residuals when only the in-phase parts of the amplitudes are fitted are small, with an rms of $12 \mu \mathrm{as}$. When the out-of-phase parts are also sought to be fitted by allowing the resonance 
parameters to be complex, the rms of residuals doubles, essentially because of a few large outof-phase residuals: about $120 \mu$ as in the prograde annual which is almost certainly due to the $S_{1}$ thermal atmospheric tide, and a similar residual in the 18.6 year prograde and about $40 \mu a s$ in the 9.3 year prograde which need to be explained. The unreduced resonance formula which involves two extra adjustable parameters provides a slightly better fit ( $\mathrm{rms}$ of residuals $21 \mu \mathrm{as}$ ) and may be used where the best fit is the only objective; however, the estimates obtained for the parameters in this formula which does not respect the sum rules are not suitable for geophysical interpretation.

\section{Concluding remarks}

Vast improvement has taken place over the past few years in the precision of nutation data obtained from VLBI observations, especially in respect of the long period (18.6 years and 9.3 years) components; and the demands placed thereby on the accuracy of the rigid Earth nutation series for the purpose of geophysical modeling have been essentially met. Parameter estimates from inverse modeling of the observed nutations have begun to yield more information about the Earth's deep interior than was available heretofore, and suggest, in particular, a closer look at the electromagnetic couplings at the CMB and the ICB. The efforts to improve forward modeling may well be helped by such insights, and are also likely to help constrain the considerable freedom which remains in the modeling of mantle anelasticity. Theoretical evaluation of atmospheric effects on nutations remains an unfinished task, as does the reduction of uncertainties in estimates of ocean tide effects including, in particular, the effect of ocean tidal currents. Finally, points of concern regarding the amplitudes of long period nutations, especially their out-of-phase parts, need to be resolved.

\section{References}

Bretagnon, P., Rocher, P. and Simon, J.-L. (1997) Theory of rotation of the rigid Earth. Astron. Astrophys., 319, pp. 305-317.

Buffett, B.A. (1992) Constraints on magnetic energy and mantle conductivity from the forced nutations of the Earth. J. Geophys. Res., 97, pp. 19,581-19,597.

Buffett, B.A., Mathews, P.M., Herring, T.A. and Shapiro, I.I. (1993) Forced nutations of the Earth: Contributions from the effects of ellipticity and rotation on the elastic deformations. J. Geophys. Res., 98, pp. 21,659-21,676.

Defraigne, P. (1997) Geophysical model of the dynamical flattening of the Earth in agreement with the precession constant. Geophys. J. Int., 130 , pp. 47-56.

Dehant, V. and Defraigne, P. (1997) New transfer functions for nutations of a non-rigid Earth. J. Geophys. Res., accepted.

Fukushima, T. (1991) Effects of geodesic precessions annual effect. Astron. Astrophys., 244, pp. L11.

Gwinn, C.R., Herring, T.A. and Shapiro, I.I. (1986) Geodesy by radio interferometry: Studies of the forced nutations of the Earth, 2. Interpretation. J. Geophys. Res., 91, pp. 4755-4765.

Herring, T.A., Buffett, B.A., Mathews, P.M. and Shapiro, I.I. (1991) Forced nutations of the Earth: Influence of inner core dynamics, III. Data analysis, J. Geophys. Res., 96, pp. 8259-8273.

Herring, T.A., Gwinn, C.R. and Shapiro, I.I. (1986) Geodesy by radio interferometry: Studies of forced nutations of the Earth, 1. Data analysis, J. Geophys. Res., 91, pp. 4745-4754.

Mathews, P.M., Buffett, B.A., Herring, T.A. and Shapiro, I.I. (1991a) Forced nutations of the Earth: Influence of inner core dynamics, I. Theory. J. Geophys. Res., 96, pp. 8219-8242.

Mathews, P.M., Buffett, B.A., Herring, T.A. and Shapiro, I.I. (1991b) Forced nutations of the Earth: Influence of inner core dynamics, II. Numerical results and comparisons. J. Geophys. Res., 96, pp. 8243-8257.

Roosbeek, F. and Dehant, V. (1997) RDAN97: An analytical development of the rigid Earth nutation series using the torque approach. Celest. Mech., submitted.

Schastok, J. (1997) A new nutation series for a more realistic model Earth. Geophys. J. Int., 130,pp. 137-150.

Simon, J.-L., Bretagnon, P., Chapront, J., Chapront-Touzé M., Francou, G. and Laskar, J. (1994) Numerical Expressions for Precession Formulae and Mean Elements for the Moon and Planets. Astron. Astrophys., 282, pp. $663-683$.

Souchay, J. and Kinoshita, H. (1996) Corrections and new developments in rigid-Earth nutation theory, I. Lunisolar influence including indirect planetary effects. Astron. Astrophys., 312, pp. 1017-1030.

Souchay, J. and Kinoshita, H. (1997) Corrections and new developments in rigid-Earth nutation theory, II. Influence of second-order geopotential and direct planetary effect. Astron. Astrophys., 318, pp. 639-652.

Wahr, J.M. (1981) The forced nutations of an elliptical, rotating, elastic, and oceanless Earth. Geophys. J. R. Astron. Soc., 64, pp. 705-727.

Wahr, J.M. and Bergen, Z. (1986) The effects of mantle anelasticity on nutations, Earth tides and tidal variations in rotation rate, Geophys. J. R. Astron. Soc., 87, pp. 633-668.

Williams, J.G. (1994) Contributions to the Earth's obliquity rate, precession, and nutation. Astran. J., 108, pp. 711-724. 